\title{
Fourier Series Coefficients for Powers of the Jacobian Elliptic Functions
}

\author{
By Ayşe Kiper
}

\begin{abstract}
The Fourier series expansion coefficients for the Jacobian elliptic functions $\operatorname{sn}^{m}(u, k)$, $\mathrm{cn}^{m}(u, k)$ and $\operatorname{dn}^{m}(u, k)$, with $m \geqslant 1$, are studied. Two-term recurrence formulae are obtained and some of the coefficients are tabulated.
\end{abstract}

1. Introduction. In the last few years, the papers written on the Jacobian elliptic functions (hereafter referred to as JEFs) have been in the direction of developing the properties of the Taylor series expansion coefficients of the functions $\operatorname{sn}(x, k)$, $\operatorname{cn}(x, k)$ and $\operatorname{dn}(x, k)$ (Schett [7], [8], [9], Wrigge [11], [12], Dumont [3], Fransén [5]). Wrigge [11] studied and obtained recurrence formulae for the Taylor series coefficients of $\operatorname{sn}^{m}(x, k)$ when $m=1$ and $m=2$. Although the Fourier series expansion for the twelve JEFs have been studied and given by several authors (Abramowitz and Stegun [1], Byrd and Friedman [2], Du Val [4], Whittaker and Watson [10]), no recurrence formula has been given for the coefficients of these series corresponding to powers of the JEFs. Those for $\mathrm{cn}^{2}(u, k)$ and $\mathrm{dn}^{2}(u, k)$ can be easily obtained by using fundamental relations and the expansion of $\operatorname{sn}^{2}(u, k)$ which is given by Whittaker and Watson [10, Art. 22.735, Ex. 5, p. 520]. In this paper, we obtain recurrence formulae for these coefficients of $\operatorname{sn}^{m}(u, k), \mathrm{cn}^{m}(u, k)$ and $\mathrm{dn}^{m}(u, k)$, with $m=1,2,3, \ldots$, in series of the same form.

2. Definitions and Preliminaries. The derivation of these formulae is based on the Fourier series for $\operatorname{sn}(u, k), \operatorname{cn}(u, k)$ and $\operatorname{dn}(u, k)$ (see (A1), (A2), (A3) in Appendix) or the following Fourier series for $\operatorname{sn}^{2}(u, k), \mathrm{cn}^{2}(u, k)$ and $\operatorname{dn}^{2}(u, k)$, depending on whether the power of the JEF to be expanded is odd or even.

$$
\begin{aligned}
& \operatorname{sn}^{2}(u, k)=\frac{K-E}{K k^{2}}-\frac{2 \pi^{2}}{K^{2} k^{2}} \sum_{n=1}^{\infty} \frac{n q^{n}}{1-q^{2 n}} \cos \frac{n \pi}{K} u, \\
& \operatorname{cn}^{2}(u, k)=\frac{E-K k^{\prime 2}}{K k^{2}}+\frac{2 \pi^{2}}{K^{2} k^{2}} \sum_{n=1}^{\infty} \frac{n q^{n}}{1-q^{2 n}} \cos \frac{n \pi}{K} u, \text { and } \\
& \operatorname{dn}^{2}(u, k)=\frac{E}{K}+\frac{2 \pi^{2}}{K^{2}} \sum_{n=1}^{\infty} \frac{n q^{n}}{1-q^{2 n}} \cos \frac{n \pi}{K} u,
\end{aligned}
$$

Received September 21, 1982; revised February 23, 1983 and July 25, 1983

1980 Mathematics Subject Classification. Primary 33A25. 
which are the periodic functions of $u$ with period $2 K$ and are valid when $|\operatorname{Im}(u / 2 K)|$ $<\operatorname{Im}\left(i K^{\prime} / K\right)$. In the above, $K$ and $E$ are the complete elliptic integrals of the first and second kind, respectively, $k$ is the modulus, $k^{\prime}=\left(1-k^{2}\right)^{1 / 2}$ the complementary modulus of the elliptic functions and the quantity $q=\exp \left(-\pi K^{\prime} / K\right)$ is referred to as the nome.

From the basic properties of $\operatorname{sn}(u, k), \operatorname{cn}(u, k)$ and $\operatorname{dn}(u, k)$ it is easily shown that

$$
\frac{d^{2}}{d u^{2}} \operatorname{sn}^{m}(u, k), \quad \frac{d^{2}}{d u^{2}} \operatorname{cn}^{m}(u, k) \text { and } \frac{d^{2}}{d u^{2}} \operatorname{dn}^{m}(u, k)
$$

with $m \geqslant 1$, successively lead to the expressions [10, Art. 22.72, Ex. 4, p. 516]:

$$
\begin{aligned}
k^{m+2} \mathrm{sn}^{m+2}(u, k)= & \frac{k^{m}}{m(m+1)} \frac{d^{2}}{d u^{2}} \operatorname{sn}^{m}(u, k)-\frac{(m-1) k^{m}}{(m+1)} \mathrm{sn}^{m-2}(u, k) \\
& +\frac{m\left(k^{2}+1\right) k^{m}}{(m+1)} \operatorname{sn}^{m}(u, k),
\end{aligned}
$$

$$
\begin{aligned}
k^{m+2} \mathrm{cn}^{m+2}(u, k)= & -\frac{k^{m}}{m(m+1)} \frac{d^{2}}{d u^{2}} \mathrm{cn}^{m}(u, k) \\
& +\frac{(m-1) k^{\prime 2} k^{m}}{(m+1)} \mathrm{cn}^{m-2}(u, k) \\
& +\frac{m\left(k^{2}-k^{\prime 2}\right) k^{m}}{(m+1)} \mathrm{cn}^{m}(u, k), \\
k^{m+2} \mathrm{dn}^{m+2}(u, k)= & -\frac{k^{m}}{m(m+1)} \frac{d^{2}}{d u^{2}} \mathrm{dn}^{m}(u, k) \\
& -\frac{(m-1) k^{\prime 2} k^{m+2}}{(m+1)} \mathrm{dn}^{m-2}(u, k) \\
& +\frac{m\left(1+k^{\prime 2}\right) k^{m+2}}{(m+1)} \operatorname{dn}^{m}(u, k),
\end{aligned}
$$

each of which expresses even (odd) powers of one of the elliptic functions in terms of even (odd) powers of the JEF of the corresponding type.

3. Derivation of Recurrence Formulae. To derive the recurrence relation for the Fourier series coefficients of the JEF $\operatorname{sn}(u, k)$ of odd (even) powers, the Fourier series for

$$
k^{m+2} \mathrm{sn}^{m+2}(u, k)
$$

and

$$
\frac{d^{2}}{d u^{2}}\left[k^{m+2} \mathrm{sn}^{m+2}(u, k)\right]
$$

are used with $m=2 r+1(m=2 r)$.

Differentiating (A1) twice and using (4) with $m=2 r+1$, the Fourier coefficients corresponding to (7) and (8) can be generated successively with the increasing values 
of $r(r=0,1,2, \ldots)$. Then, by induction, we obtain the generalization for odd powers of the JEF $\operatorname{sn}(u, k)$ as

(9) $k^{2 r+3} \mathrm{sn}^{2 r+3}(u, k)$

$$
=\frac{2 \pi}{K} \sum_{n=0}^{\infty} \frac{q^{n+1 / 2}}{1-q^{2 n+1}} \mathscr{S}_{s, n}^{(r)} \sin \frac{(2 n+1) \pi}{2 K}, \quad r=-1,0,1, \ldots,
$$

where

$$
\left\{\begin{array}{r}
\mathscr{S}_{s, n}^{(-2)}=0, \\
\mathscr{S}_{s, n}^{(-1)}=1, \\
\mathscr{S}_{s, n}^{(r)}=\frac{1}{2(r+1)}\left[\left((2 r+1)\left(1+k^{2}\right)-\frac{(2 n+1)^{2} \pi^{2}}{4(2 r+1) K^{2}}\right) \mathscr{S}_{s, n}^{(r-1)}\right. \\
\left.-2 r k^{2} \mathscr{S}_{s, n}^{(r-2)}\right], \\
r=0,1,2, \ldots
\end{array}\right),
$$

with the subscript " $s$ " denoting the function $\operatorname{sn}(u, k)$.

In the case of even powers of $\operatorname{sn}(u, k)$, this relation is deduced from the Fourier coefficients of the series (7) and (8), generated successively with $m=2 r$ ( $r=$ $1,2,3, \ldots)$, by making use of (4). The process initially requires the differentiation of (1) twice with respect to $x$, which leads to

$$
\begin{aligned}
k^{2 r+2} & \operatorname{sn}^{2 r+2}(u, k) \\
= & \mathscr{P}_{s}^{(r)}+\frac{2 \pi^{2}}{K^{2}} \sum_{n=1}^{\infty} \frac{n q^{n}}{1-q^{2 n}} \mathscr{R}_{s, n}^{(r)} \cos \frac{n \pi}{K} u, \quad r=0,1,2, \ldots,
\end{aligned}
$$

where

$$
\left\{\begin{array}{l}
\mathscr{P}_{s}^{(-1)}=1 \\
\mathscr{P}_{s}^{(0)}=1-E / K \\
\mathscr{P}_{s}^{(r)}=\frac{1}{(2 r+1)}\left[2 r\left(k^{2}+1\right) \mathscr{P}_{s}^{(r-1)}-(2 r-1) k^{2} \mathscr{P}_{s}^{(r-2)}\right]
\end{array}\right.
$$

$$
r=1,2,3, \ldots
$$

and

$$
\left\{\begin{array}{l}
\mathscr{R}_{s, n}^{(-1)}=0, \\
\mathscr{R}_{s, n}^{(0)}=-1, \\
\mathscr{R}_{s, n}^{(r)}=\frac{1}{(2 r+1)}\left[\left(2 r\left(k^{2}+1\right)-\frac{n^{2} \pi^{2}}{2 r K^{2}}\right) \mathscr{R}_{s, n}^{(r-1)}\right. \\
\left.-(2 r-1) k^{2} \mathscr{R}_{s, n}^{(r-2)}\right], \\
r=1,2, \ldots
\end{array}\right\},
$$


Following a similar procedure, the two-term recurrence relations for the coefficients of odd and even powers of the remaining two types of JEFs can be found as (14)

$$
\begin{aligned}
& k^{2 r+3} \mathrm{cn}^{2 r+3}(u, k) \\
& =\frac{2 \pi}{K} \sum_{n=0}^{\infty} \frac{q^{n+1 / 2}}{1+q^{2 n+1}} \mathscr{S}_{c, n}^{(r)} \cos \frac{(2 n+1) \pi}{2 K} u, \quad r=-1,0,1,2, \ldots
\end{aligned}
$$

with

$$
\left\{\begin{array}{r}
\mathscr{S}_{c, n}^{(-2)}=0, \\
\mathscr{S}_{c, n}^{(-1)}=1, \\
\mathscr{S}_{c, n}^{(r)}=\frac{1}{2(r+1)}\left[\left((2 r+1)\left(k^{2}-k^{2}\right)+\frac{(2 n+1)^{2} \pi^{2}}{4(2 r+1) K^{2}}\right) \mathscr{S}_{c, n}^{(r-1)}\right. \\
\left.+2 r k^{2} k^{\prime 2} \mathscr{S}_{c, n}^{(r-2)}\right], \\
r=0,1,2, \ldots
\end{array}\right\},
$$

(16) $k^{2 r+2} \mathrm{cn}^{2 r+2}(u, k)$

$$
=\mathscr{P}_{c}^{(r)}+\frac{2 \pi^{2}}{K^{2}} \sum_{n=1}^{\infty} \frac{n q^{n}}{1-q^{2 n}} \mathscr{R}_{c, n}^{(r)} \cos \frac{n \pi}{K} u, \quad r=0,1,2, \ldots,
$$

with

$$
\begin{aligned}
& \left\{\begin{array}{l}
\mathscr{P}_{c}^{(-1)}=1, \\
\mathscr{P}_{c}^{(0)}=E / K-k^{\prime 2}, \\
\mathscr{P}_{c}^{(r)}=\frac{1}{(2 r+1)}\left[2 r\left(k^{2}-k^{\prime 2}\right) \mathscr{P}_{c}^{(r-1)}+(2 r-1) k^{2}{k^{\prime}}^{2} \mathscr{P}_{c}^{(r-2)}\right],
\end{array}\right. \\
& r=1,2,3, \ldots,
\end{aligned}
$$

and

$$
\left\{\begin{array}{l}
\mathscr{R}_{c, n}^{(-1)}=0, \\
\mathscr{R}_{c, n}^{(0)}=1, \\
\mathscr{R}_{c, n}^{(r)}=\frac{1}{(2 r+1)}\left[\left(2 r\left(k^{2}-k^{\prime 2}\right)+\frac{n^{2} \pi^{2}}{2 r K^{2}}\right) \mathscr{R}_{c, n}^{(r-1)}\right. \\
\left.+(2 r-1) k^{2} k^{\prime 2} \mathscr{R}_{c, n}^{(r-2)}\right], \\
r=1,2, \ldots
\end{array}\right\}, \quad n=1,2, \ldots .
$$


(19) $k^{2 r+3} \mathrm{dn}^{2 r+3}(u, k)$

$$
=\mathscr{Q}_{d}^{(r)}+\frac{2 k \pi}{K} \sum_{n=0}^{\infty} \frac{q^{n+1}}{1+q^{2(n+1)}} \mathscr{S}_{d, n}^{(r)} \cos \frac{(n+1) \pi}{K} u, \quad r=-1,0,1, \ldots,
$$

with

(20)

$$
\left\{\begin{array}{l}
\mathscr{Q}_{d}^{(-2)}=0 \\
\mathscr{Q}_{d}^{(-1)}=k \pi / 2 K \\
\mathscr{Q}_{d}^{(r)}=\frac{k^{2}}{2(r+1)}\left[(2 r+1)\left(1+k^{\prime 2}\right) \mathscr{Q}_{d}^{(r-1)}-2 r k^{2} k^{\prime 2} \mathscr{Q}_{d}^{(r-2)}\right]
\end{array}\right.
$$$$
r=0,1,2, \ldots,
$$

and

(21)

$$
\left\{\begin{array}{r}
\mathscr{S}_{d, n}^{(-2)}=0, \\
\mathscr{S}_{d, n}^{(-1)}=1, \\
\mathscr{S}_{d, n}^{(r)}=\frac{k^{2}}{2(r+1)}\left[\left((2 r+1)\left(1+k^{\prime 2}\right)+\frac{(n+1)^{2} \pi^{2}}{(2 r+1) K^{2}}\right) \mathscr{S}_{d, n}^{(r-1)}\right. \\
\left.-2 r k^{2} k^{\prime 2} \mathscr{S}_{d, n}^{(r-2)}\right], \\
r=0,1,2, \ldots
\end{array}\right],
$$

(22) $k^{2 r+2} \mathrm{dn}^{2 r+2}(u, k)$

$$
=\mathscr{P}_{d}^{(r)}+\frac{2 \pi^{2} k^{2}}{K^{2}} \sum_{n=1}^{\infty} \frac{n q^{n}}{1-q^{2 n}} \mathscr{R}_{d, n}^{(r)} \cos \frac{n \pi}{K} u, \quad r=0,1,2, \ldots,
$$

with

(23) $\left\{\begin{array}{l}\mathscr{P}_{d}^{(-1)}=1, \\ \mathscr{P}_{d}^{(0)}=E k^{2} / K, \\ \mathscr{P}_{d}^{(r)}=\frac{k^{2}}{(2 r+1)}\left[2 r\left(1+k^{\prime 2}\right) \mathscr{P}_{d}^{(r-1)}-(2 r-1) k^{2} k^{\prime 2} \mathscr{P}_{d}^{(r-2)}\right],\end{array}\right.$ 
and

$$
\left\{\begin{array}{l}
\mathscr{R}_{d, n}^{(-1)}=0, \\
\mathscr{R}_{d, n}^{(0)}=1, \\
\mathscr{R}_{d, n}^{(r)}=\frac{1}{(2 r+1)}\left[\left(2 r\left(1+k^{\prime 2}\right)+\frac{n^{2} \pi^{2}}{2 r K^{2}}\right) \mathscr{R}_{d, n}^{(r-1)}\right. \\
\left.+(2 r-1) k^{2} k^{\prime 2} \mathscr{R}_{d, n}^{(r-2)}\right], \\
r=1,2, \ldots
\end{array}\right\}, \quad n=1,2, \ldots .
$$

In this paper, the constant term and the expansion coefficients of the Fourier series for odd (even) powers of JEFs are denoted by $\mathscr{Q}(\mathscr{P})$ and $\mathscr{S}(\mathscr{R})$, respectively.

4. Results. In this section we give numerical results for some of these coefficients. It is clear from (9), (10), ., (24) that the accuracy of the results depends strongly on that of $K, E$ and $q$. For this reason the latter quantities were computed to ten decimal places prior to doing the computations of the coefficients themselves. All of the computations were done at Middle East Technical University (Ankara) on the Burroughs 6900 using single-precision FORTRAN.

TABle 1

Values of $\mathscr{P}_{s}^{(r)}, \mathscr{P}_{c}^{(r)}, \mathscr{P}_{d}^{(r)}, \mathscr{Q}_{d}^{(r)}$ for $k^{2}=0.7$

\begin{tabular}{|c|c|c|c|c|}
\hline & $\mathrm{r}=-1$ & $\mathrm{r}=0$ & $r=1$ & $r=2$ \\
\hline$p_{s}^{(r)}$ & 1 & 0.4017092494 & 0.2219371494 & 0.1331166385 \\
\hline$p_{c}^{(r)}$ & 1 & 0.2982907506 & 0.1495442002 & 0.0854387786 \\
\hline$p_{d}^{(r)}$ & 1 & 0.4188035254 & 0.2050741387 & 0.1123555021 \\
\hline $2_{d}^{(r)}$ & 0.6332494173 & 0.2881284849 & 0.1501038588 & 0.0855921680 \\
\hline
\end{tabular}


TABLE 2

Values of $\frac{2 \pi}{K} \frac{q^{n+1 / 2}}{1-q^{2 n+1}} \mathscr{S}_{s, n}^{(r)}$ for $k^{2}=0.7$

\begin{tabular}{|c|c|c|c|c|c|c|}
\hline $\mathrm{n}$ & $r=-1$ & $r=0$ & & $r=1$ & $r=$ & \\
\hline 0 & 0.8941894747 & 0.50393 & 65582 & 0.3054955347 & 0.19178 & 13668 \\
\hline 1 & 0.0618244070 & -0.10682 & 56608 & -0.1119438256 & -0.08949 & 65338 \\
\hline 2 & 0.0046157482 & -0.02912 & 90657 & -0.0039904996 & 0.00984 & 53677 \\
\hline 3 & 0.0003447492 & -0.00454 & 55761 & 0.0047167163 & 0.00438 & 99563 \\
\hline 4 & 0.0000257403 & -0.00057 & 55223 & 0.0014826451 & 0.00007 & 57350 \\
\hline 5 & 0.0000019232 & -0.00006 & 50205 & 0.0002920089 & -0.00023 & 06795 \\
\hline 6 & 0.0000001436 & -0.00000 & 68313 & 0.0000463537 & -0.00008 & 07339 \\
\hline 7 & 0.0000000107 & -0.00000 & 06823 & 0.0000064552 & -0.00001 & 82715 \\
\hline 8 & 0.0000000008 & -0.00000 & 00657 & 0.0000008218 & -0.00000 & 33403 \\
\hline 9 & 0 & -0.00000 & 00061 & 0.0000000979 & -0.00000 & 05335 \\
\hline 10 & 0 & -0.00000 & 00006 & 0.0000000111 & -0.00000 & 00774 \\
\hline 11 & 0 & 0 & & 0 & -0.00000 & 00105 \\
\hline 12 & 0 & 0 & & 0 & -0.00000 & 00013 \\
\hline
\end{tabular}




\section{TABLE 3}

Values of $\frac{2 \pi^{2}}{K^{2}} \frac{n q^{n}}{1-q^{2 n}} \mathscr{R}_{s, n}^{(r)}$ for $k^{2}=0.7$

\begin{tabular}{|c|c|c|c|c|c|c|c|}
\hline $\mathrm{n}$ & $r=0$ & $r=1$ & & $r=2$ & & $r=3$ & \\
\hline 1 & -0.3442177075 & -0.25865 & 34388 & -0.17756 & 25892 & -0.11971 & 97943 \\
\hline 2 & $-0.05113 \quad 39471$ & 0.02016 & 23245 & 0.03965 & 68027 & 0.03905 & 00051 \\
\hline 3 & -0.0057286101 & 0.01319 & 78635 & 0.00674 & 60642 & -0.00008 & 14475 \\
\hline 4 & -0.0005704927 & 0.00283 & 94654 & -0.00110 & 39285 & -0.00206 & 46556 \\
\hline 5 & -0.0000532626 & 0.00044 & 81727 & -0.00065 & 18249 & -0.00028 & 48240 \\
\hline 6 & -0.0000047738 & 0.00006 & 02236 & -0.00016 & 44904 & 0.00005 & 32787 \\
\hline 7 & $-0.00000 \quad 04160$ & 0.00000 & 73131 & -0.00003 & 09355 & 0.00003 & 39680 \\
\hline 8 & -0.0000000355 & 0.00000 & 08277 & -0.00000 & 49284 & 0.00000 & 96135 \\
\hline 9 & -0.0000000030 & 0.00000 & 00889 & -0.00000 & 07030 & 0.00000 & 20379 \\
\hline 10 & -0.0000000002 & 0.00000 & 00092 & -0.00000 & 00925 & 0.00000 & 03655 \\
\hline 11 & 0 & 0.00000 & 00009 & -0.00000 & 00115 & 0.00000 & 00585 \\
\hline 12 & 0 & 0.00000 & 00001 & -0.00000 & 00014 & 0.00000 & 00086 \\
\hline 13 & 0 & 0 & & -0.00000 & 00002 & 0.00000 & 00012 \\
\hline
\end{tabular}


TABLE 4

Values of $\frac{2 \pi}{K} \frac{q^{n+1 / 2}}{1+q^{2 n+1}} \mathscr{S}_{c, n}^{(r)}$ for $k^{2}=0.7$

\begin{tabular}{|c|c|c|c|c|c|}
\hline $\mathrm{n}$ & $r=-1$ & $r=0$ & $r=1$ & $r=2$ & \\
\hline 0 & 0.7698988143 & 0.3745034335 & 0.0493899502 & -0.03502 & 40397 \\
\hline 1 & 0.0617729084 & 0.1715982308 & 0.1187201550 & 0.03595 & 27848 \\
\hline 2 & 0.0046157267 & 0.0339754433 & 0.0502565433 & 0.03598 & 74242 \\
\hline 3 & 0.0003447492 & 0.0049075626 & 0.0129158150 & 0.01570 & 32559 \\
\hline 4 & 0.0000257493 & 0.0006025591 & 0.0025080590 & 0.00463 & 09588 \\
\hline 5 & 0.0000019232 & 0.0000670399 & 0.0004071579 & 0.00106 & 70931 \\
\hline 6 & 0.0000001436 & $0.00000 \quad 69821$ & 0.0000584103 & 0.00020 & 69905 \\
\hline 7 & $0.00000 \quad 00107$ & $0.00000 \quad 06936$ & 0.0000076569 & 0.00003 & 53530 \\
\hline 8 & 0.0000000008 & 0.0000000665 & 0.0000009372 & 0.00000 & 54754 \\
\hline 9 & 0 & 0.0000000062 & $0.00000 \quad 01087$ & 0.00000 & 07848 \\
\hline 10 & 0 & 0.0000000006 & $0.00000 \quad 00121$ & 0.00000 & 01056 \\
\hline 11 & 0 & 0 & 0.0000000013 & 0.00000 & 00135 \\
\hline 12 & 0 & 0 & 0 & 0.00000 & 00017 \\
\hline
\end{tabular}


TABLE 5

Values of $\frac{2 \pi^{2}}{K^{2}} \frac{n q^{n}}{1-q^{2 n}} \mathscr{R}_{c, n}^{(r)}$ for $k^{2}=0.7$

\begin{tabular}{|c|c|c|c|c|c|}
\hline $\mathrm{n}$ & $r=0$ & $r=1$ & $r=2$ & $r=$ & \\
\hline 1 & 0.3442177075 & 0.2232513517 & 0.1403903977 & 0.08928 & 10400 \\
\hline 2 & 0.0511339471 & 0.0917498504 & 0.0778509810 & 0.05744 & 39671 \\
\hline 3 & 0.0057286101 & $0.02121 \quad 79177$ & 0.0293905060 & 0.02769 & 09444 \\
\hline 4 & 0.0005704927 & 0.0036381551 & $0.00790 \quad 54300$ & 0.01015 & 70883 \\
\hline 5 & 0.0000532626 & 0.0005227403 & 0.0016712836 & 0.00293 & 09898 \\
\hline 6 & 0.0000047738 & $0.00006 \quad 69069$ & 0.0002979775 & 0.00069 & 74589 \\
\hline 7 & 0.0000004160 & 0.0000078954 & 0.0000469044 & 0.00014 & 26585 \\
\hline 8 & 0.0000000355 & 0.0000008774 & 0.0000067187 & 0.00002 & 58950 \\
\hline 9 & $0.00000 \quad 00030$ & $0.00000 \quad 00931$ & $0.00000 \quad 08941$ & 0.00000 & 42718 \\
\hline 10 & $0.00000 \quad 00002$ & 0.0000000095 & $0.00000 \quad 01122$ & 0.00000 & 06519 \\
\hline 11 & 0 & 0.0000000009 & 0.0000000134 & 0.00000 & 00933 \\
\hline
\end{tabular}


TABLE 6

Values of $\frac{2 k \pi}{K} \frac{q^{n+1}}{1+q^{2(n+1)}} \mathscr{S}_{d, n}^{(r)}$ for $k^{2}=0.7$

\begin{tabular}{|c|c|c|c|c|c|}
\hline $\mathrm{n}$ & $r=-1$ & $r=0$ & $r=1$ & $r=$ & \\
\hline 0 & 0.5577980123 & $0.70115 \quad 74837$ & 0.5312643400 & 0.36256 & 72995 \\
\hline 1 & 0.0418930129 & 0.1534557231 & 0.1837030417 & 0.16355 & 79089 \\
\hline 2 & 0.0031290836 & 0.0240097302 & 0.0450407061 & 0.05347 & 67638 \\
\hline 3 & 0.0002337111 & 0.0031053557 & 0.0087436281 & 0.01380 & 62322 \\
\hline 4 & 0.0000174559 & 0.0003579368 & 0.0014391288 & 0.00297 & 99203 \\
\hline 5 & $0.00000 \quad 13038$ & 0.0000382363 & 0.0002099960 & 0.00055 & 97050 \\
\hline 6 & $0.00000 \quad 00974$ & 0.0000038712 & 0.0000279900 & 0.00009 & 41773 \\
\hline 7 & $0.00000 \quad 00073$ & $0.00000 \quad 03766$ & 0.0000034785 & 0.00001 & 45042 \\
\hline 8 & 0.0000000005 & 0.0000000355 & $0.00000 \quad 04090$ & 0.00000 & 20779 \\
\hline 9 & 0 & 0.0000000033 & $0.00000 \quad 00460$ & 0.00000 & 02804 \\
\hline 10 & 0 & 0.0000000003 & 0.0000000050 & 0.00000 & 00360 \\
\hline 11 & 0 & 0 & $0.00000 \quad 00005$ & 0.00000 & 00044 \\
\hline 12 & 0 & 0 & 0 & 0.00000 & 00005 \\
\hline
\end{tabular}




\section{TABLE 7}

Values of $\frac{2 \pi^{2} k^{2}}{K^{2}} \frac{n q^{n}}{1-q^{2 n}} \mathscr{R}_{d, n}^{(r)}$ for $k^{2}=0.7$

\begin{tabular}{|c|c|c|c|c|c|}
\hline $\mathrm{n}$ & $r=0$ & $r=1$ & $r=2$ & $r=$ & \\
\hline 1 & 0.2409523953 & 0.2105931683 & 0.1489496005 & 0.09975 & 69304 \\
\hline 2 & 0.0357937630 & 0.0599908071 & $0.05976 \quad 15801$ & 0.04944 & 44044 \\
\hline 3 & 0.0040100271 & 0.0120809910 & 0.0171614413 & 0.01801 & 61237 \\
\hline 4 & 0.0003993449 & 0.0019504208 & 0.0038874943 & 0.00520 & 29241 \\
\hline 5 & 0.0000372838 & 0.0002718019 & 0.0007395528 & 0.00125 & 22174 \\
\hline 6 & $0.00000 \quad 33417$ & 0.0000341879 & 0.0001233026 & 0.00026 & 21117 \\
\hline 7 & $0.00000 \quad 02912$ & $0.00000 \quad 39911$ & 0.0000185641 & 0.00004 & 88009 \\
\hline 8 & 0.0000000249 & 0.0000004403 & 0.0000025786 & 0.00000 & 82678 \\
\hline 9 & $0.00000 \quad 00021$ & 0.0000000465 & $0.00000 \quad 03357$ & 0.00000 & 12954 \\
\hline 10 & 0.0000000002 & 0.0000000047 & $0.00000 \quad 00414$ & 0.00000 & 01901 \\
\hline 11 & 0 & 0.0000000005 & 0.0000000049 & 0.00000 & 00264 \\
\hline 12 & 0 & 0 & 0.0000000006 & 0.00000 & 00035 \\
\hline
\end{tabular}

5. Acknowledgements. I am greatly indebted to the referee for his valuable suggestions, which improved the text.

\section{Appendix}

Fourier series for the Jacobian elliptic functions [2, pp. 304, 305]:

(A1) $\operatorname{sn}(u, k)=\frac{2 \pi}{k K} \sum_{n=0}^{\infty} \frac{q^{n+1 / 2}}{1-q^{2 n+1}} \sin \frac{(2 n+1) \pi}{2 K} u$,

$\left[|\operatorname{Im}(u / K)|<\operatorname{Im}\left(i K^{\prime} / K\right)\right]$, 
(A2) $\operatorname{cn}(u, k)=\frac{2 \pi}{k K} \sum_{n=0}^{\infty} \frac{q^{n+1 / 2}}{1+q^{2 n+1}} \cos \frac{(2 n+1) \pi}{2 K} u$

$$
\left[|\operatorname{Im}(u / K)|<\operatorname{Im}\left(i K^{\prime} / K\right)\right],
$$

$$
\begin{aligned}
\operatorname{dn}(u, k)=\frac{\pi}{2 K}+\frac{2 \pi}{K} \sum_{n=0}^{\infty} \frac{q^{n+1}}{1+q^{2(n+1)}} \cos \frac{(n+1) \pi}{K} u, \\
\quad\left[|\operatorname{Im}(u / K)|<\operatorname{Im}\left(i K^{\prime} / K\right)\right],
\end{aligned}
$$

where the periods for (A1) and (A2) are $4 K$ and that for (A3) is $2 K$.

Department of Computer Sciences

Middle East Technical University

Ankara, Turkey

1. M. Abramowitz \& I. A. Stegun, Handbook of Mathematical Functions with Formulas, Graphs, and Mathematical Tables, Nat. Bur. Standards, Appl. Math. Series No. 55, December 1954. (Also: Dover, New York, 1968.)

2. P. F. BYRD \& M. D. Friedman, Handbook of Elliptic Integrals for Engineers and Scientists, 2nd ed., Springer-Verlag, Berlin, 1971.

3. D. Dumont, "A combinatorial interpretation for the Schett recurrence on the Jacobian elliptic functions," Math. Comp., v. 33, 1979, pp. 1293-1297.

4. P. Du VAL, Elliptic Functions and Elliptic Curves, London Mathematical Society Lecture Note Series 9, Cambridge Univ. Press, Cambridge, 1973.

5. A. Fransén, "Conjectures on the Taylor series expansion coefficients of the Jacobian elliptic function $\operatorname{sn}(x, k)$, , Math. Comp., v. 37, 1981, pp. 475-494.

6. R. G. LANGeBartel, "Fourier expansions of rational fractions of elliptic integrals and Jacobian elliptic functions," SIAM J. Math. Anal., v. 11, 1980, pp. 506-513.

7. A. SchetT, "Properties of the Taylor series expansion coefficients of the Jacobian elliptic functions," Math. Comp., v. 30, 1976, pp. 143-147.

8. A. Schetr, Addendum to "Properties of the Taylor series expansion coefficients of the Jacobian elliptic functions," Math. Comp., v. 31, 1977, Microfiche supplement.

9. A. SchetT, "Recurrence formula of the Taylor series expansion coefficients of the Jacobian elliptic functions," Math. Comp., v. 31, 1977, pp. 1003-1005.

10. E. T. Whittaker \& G. N. Watson, Modern Analysis, Cambridge Univ. Press, Cambridge, 1962.

11. S. WrIGGE, "Calculation of the Taylor series expansion coefficients of the Jacobian elliptic function $\operatorname{sn}(x, k)$, , Math. Comp., v. 36, 1981, pp. 555-564.

12. S. WRIGGE, "A note on the Taylor series expansion coefficients of the Jacobian elliptic function $\operatorname{sn}(x, k)$, Math. Comp., v. 37, 1981, pp. 495-497. 